\title{
Utber die gegenseitigen Störungen zweier einander nahekommenden kleinen Planeten.
}

Von Dr. Elis Strömgren.

In A. N. 3916 hat Riem die Aufmerksamkeit auf eine im Anfang dieses Jahres stattfindende Annäherung der Planeten ( 366 ) Vincentina und (386) Siegena gelenkt. Nach den Angaben yon Riem beträgt die Minimaldistanz 0.048 astronomische Einheiten. Obschon es nicht anzunehmen war, daß diese Annäherung groß genug ist, um mit plausiblen Annahmen für die Massen eine merkbare gegenseitige Störung bewirken $z \mathrm{u}$ können, habe ich mir doch vorgenommen, den Fall etwas näher zu untersuchen, um der Frage von Proximitäten kleiner Planeten überhaupt näher zu treten.

Schon vorher (A.N. 3827 ) habe ich einen ähnlichen Fall, die Annäherung des Kometen I 902 III an Merkur behandelt. Während der kurzen Zeit, wo hier eine merkbare Störung hervorgerufen werden konnte, ließen sich die Koordinatendifferenzen des störenden und des gestörten Körpers linear nach der Zeit ausdrücken. *). Der dann folgende Ausdruck für den inversen Wert der dritten Potenz der Distanz der beiden Körper enthielt also die zweite Potenz von $t$, und die auszufuhrenden Integrationen erhielten nach einer kleinen Transformation die folgenden Formen: $\iint \frac{\mathrm{d} \tau^{2}}{\left(\mathrm{r}+\tau^{2}\right)^{3 / 3}}$ und $\iint \frac{\tau \mathrm{d} \tau^{2}}{\left(\mathrm{r}+\tau^{2}\right)^{3 / 2}}$, Integrationen, die sich ja sofort in geschlossener Form ausführen lassen.

$$
\begin{aligned}
& \frac{\mathrm{d} x}{\mathrm{~d} t}=\frac{k}{\sqrt{p}} \sin a \cos \left(v+A^{\prime}\right)+\frac{k}{\sqrt{p}} e \sin a \cos A^{\prime}, \quad \frac{\mathrm{d}^{2} x}{\mathrm{~d} t^{2}}=-\frac{k^{2} x}{r^{3}}, \\
& \frac{\mathrm{d}^{3} x}{\mathrm{~d} t^{3}}=-\frac{k^{3} V \bar{p}}{r^{4}} \sin a \cos \left(v+A^{\prime}\right)+\frac{2 k^{3}}{r^{3}} \frac{e}{\sqrt{p}} \sin a \sin \left(v+A^{\prime}\right) \sin v, \\
& \frac{\mathrm{d}^{4} x}{\mathrm{~d} t^{4}}=+\frac{k^{4} p}{r^{6}} \sin a \sin \left(v+A^{\prime}\right)+\frac{4 k^{4} e}{r^{5}} \sin a \cos \left(v+A^{\prime}\right) \sin v+\frac{2 k^{4} e}{r^{5}} \sin a \sin \left(2 v+A^{\prime}\right)-\frac{6 k^{4} e^{2}}{r^{4} p} \sin a \sin \left(v+A^{\prime}\right) \sin 2 v,
\end{aligned}
$$

u. s. w. und für $\frac{\mathrm{d} y}{\mathrm{~d} t}, \frac{\mathrm{d} z}{\mathrm{~d} t}$ etc. ganz entsprechende Ausdrücke, wo nur $\sin a, A^{\prime}$, gegen $\sin b, B^{\prime}$ und $\sin c, C^{\prime}$ vertauscht sind. Mit Hilfe der Maclaurinschen Reihe

$$
x=x_{0}+\frac{t}{\mathrm{I} !} \cdot \frac{\mathrm{d} x_{0}}{\mathrm{~d} t}+\frac{t^{2}}{2 !} \cdot \frac{\mathrm{d}^{2} x_{0}}{\mathrm{~d} t^{2}}+\cdots
$$

erhalten wir also die Koordinaten der beiden Körper nach Potenzen der Zeit entwickelt.

Für die zwei in Frage stehenden Planeten lauten die Elemente (Veröff. R. I. 22):

In solchen Fällen, wo ein Komet einem störenden Planeten sehr nahe kommt, liegen die Verhältnisse für eine derartige Berechnung der Störungen besonders günstig, da die Verschiedenheit der Bahnelemente der zwei-Körper zur Folge hat, daß die Zeit der Annäherung sehr kurz wird. Ganz andere Fälle können bei gegenseitigen Annäherungen von kleinen Planeten eintreten, da hier die Dauer der Annäherung beliebig groß werden kann; und wir haben überhaupt nur den sehr kleinen Massen zu verdanken, daß nicht derartige Störungen in unserm System eine große Rolle spielen.

Wir wollen die Behandlung des Problems so zurechtlegen, daß die Entwickelungen allgemein diesen schwierigeren Bedingungen entsprechen. Wir begnügen uns in der Entwickelung der Koordinaten nicht damit, nur die erste Potenz der Zeit zu berücksichtigen.

Aus den bekannten Gleichungen

$$
\begin{aligned}
& x=r \sin a \sin \left(v+A^{\prime}\right) \\
& y=r \sin b \sin \left(v+B^{\prime}\right) \\
& z=r \sin c \sin \left(v+C^{\prime}\right)
\end{aligned}
$$

Epoche Igo4 März 24.0

(366)

$$
\begin{aligned}
& M=241^{\circ} 10^{\circ} 18.0 \\
& \omega=3145^{8} 45.5 \\
& \delta=3475048.2 \\
& i=103522.2 \\
& \varphi=3^{27} 2.7 \\
& \mu=63^{6.213}
\end{aligned}
$$$$
\log a=0.497603
$$

Mittl. Äqu. 1900.0

$$
\begin{aligned}
& M_{1}=142^{\circ} 27^{\prime} 47^{\prime \prime} \mathrm{I} \\
& \omega_{1}=2 \text { I } 72349.6 \\
& \delta_{1}=167 \circ 37.3 \\
& i_{1}=201544.5 \\
& \varphi_{1}=935 \text { I.4 } \\
& \mu_{1}=7 \times 8: 859 \\
& \log a_{1}=0.46224^{2}
\end{aligned}
$$

*) Nach der Publikation des Aufsatzes in A. N. $\mathbf{3 8 2 7}$ wurde ich durch Herrn Prof. Harzer darauf aufmerksam gemacht, daß sich bei Laplace (Mécanique Céleste, Livre IX, ch. II) eine im Prinzip ähnliche Überlegung vorfindet. 
und hieraus ergibt sich mit Hilfe von (2), wenn $t=0$ auf r904 Jan. 2 I.5 gelegt wird:

$$
\begin{aligned}
& x=-3.199964-0.045318(t / 30)+0.012 \times 5^{8}(t / 30)^{2}+0.0001036(t / 30)^{3}-0.00000622(t / 30)^{4} \cdots \\
& y=+0.685527-0.270907+0.002604+0.0003332+0.00000336 \\
& z=-0.000657-0.051296 \quad+0.000003 \quad+0.0000650 \quad+0.00000037 \\
& x_{1}=-3.168093-0.085677 \quad+0.012297 \quad-0.0000063 \quad-0.00000638 \\
& y_{1}=+0.721908-0.237030 \quad-0.002802 \quad+0.0003334 \quad-0.00000329 \\
& z_{1}=+0.003202+0.092373 \quad-0.000012 \quad-0.0001194 \quad+0.0000017 \mathrm{I} \\
& x_{1}-x=+0.031871-0.040359+0.000139 \quad-0.0001099 \quad-0.00000016 \\
& y_{1}-y=+0.03638 \mathrm{I}+0.033877 \quad-0.000198 \quad+0.0000002 \quad-0.00000665 \\
& z_{1}-z=+0.003859+0.143669 \quad-0.000015 \quad-0.0001844 \quad+0.00000134
\end{aligned}
$$

wo ich die Entwickelung nach Potenzen von $t / 30$ statt $t$ angesetzt habe, um die Zahl der Dezimalen etwas zu beschränken. Für die gegenseitige Distanz erhalten wir:

$$
\rho^{2}=0.00235418+0.0010012(t / 30)+0.0234114(t / 30)^{2}-0.00003731(t / 30)^{3}-0.00004452(t / 30)^{4} \cdots
$$

eine Gleichung, die durch die Substitution

in die folgende übergeführt wird:

$$
(t / 30)=[9.500 \times 96] \tau-0.0213814
$$

$$
\rho^{2}=0.00234348\left(1+\tau^{2}-0.0004527 \tau^{3}-0.0001103 \tau^{4} \cdots\right)
$$

wo die erste Potenz der Zeit fehlt und $\tau=0$ mit dem Moment der größten Annäherung zusammenfältt. Für die kleinste Distanz erhalten wir $\rho_{0}=0.048409$.

Wenn es jetzt gilt $1 / \varrho^{3}$ in eine Reihe zu entwickeln, sieht man sofort, daß eine reine Entwickelung nach $\tau$ nur dann konvergent wird, wenn $\tau^{2}<I$, d. i. für eine Zeit, die von dem Moment der größten Annäherung um nur 9.5 Tage verschieden ist. Beim Kometen 1902 III lag die Grenze noch niedriger, bei $2 / 5$ Tag.

Hier ist der springende Punkt, wodurch sich unser Fall ron einer reinen Entwickelung nach Potenzen der Zeit

$$
\frac{\mathrm{I}}{\rho^{3}}=\frac{\mathrm{I}}{0.00011344^{6}}\left(\frac{\mathrm{I}}{\left(\mathrm{I}+\tau^{2}\right)^{8 / 2}}+0.000679 \mathrm{I} \cdot \frac{\tau^{3}}{\left(1+\tau^{2}\right)^{5 / 2}}+0.0002855 \cdot \frac{\tau^{4}}{\left(1+\tau^{2}\right)^{5 / 2}} \cdots\right)
$$

eine Reihe, wo die drei ersten Glieder für +30 Tage gegen den direkt berechneten Wert von $\varrho^{3}, 0.0043594$, einen Fehler von nur -0.0000002 ubrig lassen.

Die ganze Sache hängt natürlich davon ab, daß in den Differenzen der Koordinaten, $\left(x_{1}-x\right)$ etc., wegen der Annäherung der zwei Körper die Glieder nullten Grades in bezug auf die Zeit von derselben Größenordnung wie die des ersten Grades ausfallen, wenn wir für die Zeit eine den Bedingungen des Problems entsprechende Einheit gewählt haben.

Aus ganz demselben Grunde erklärt sich auch z. B., $\mathrm{da} B$ in (4), (6) und (7) die mit $\tau^{3}$ und $\tau^{4}$ multiplizierten Glieder von derselben Größenordnung sind. Die danach folgenden Glieder werden von einer höheren Ordnung sein.

Für die Ausführung der Berechnung der Störungen bin ich in bezug auf die Massen der zwei in Frage kommenden Planeten den Anweisungen Bauschingers (Tabellen zur Geschichte und Statistik der kleinen Planeten, Tafel X) gefolgt. Ich habe also für (386) die größere Masse angenommen und beschränke mich auf die Berechnung der von diesem unterscheidet. Während die Entwickelungen nach Potenzen der Zeit für die Koordinaten der betreffenden Körper für relativ sehr lange Zeiten konvergent und zwar rasch konvergent sind (wir erhalten z. B., wie der Vergleich mit direkt gerechneten Werten zeigt, aus (3) die Koordinaten mit Hilfe der Glieder bis $\tau^{4}$ für 30 Tage auf die fünfte Dezimale genau), so hört die entsprechende Entwickelung von $1 / \varrho^{3}$ schon mit weniger als ro Tagen auf überhaupt zu konvergieren.

Wenn wir aber in (6) den Faktor $\left(1+\tau^{2}\right)$ herausdividieren und dann die Parenthese entwickeln, erhalten wir

Planeten in der Bewegung des Planeten (366) bewirkten Störungen. Um ein erläuterndes Beispiel zu erhalten, wähle ich die Methode der Variation der Elemente, obschon in diesem Falle die viel einfachere Berechnung der Störungen in den rechtwinkligen Koordinaten genügen würde, um das schließlich erlangte negative Resultat zu geben. Was die Genauigkeit der Rechnung betrifft, habe ich mich bei der Ausführung dieses Beispiels nicht nach dem speziellen Falle gerichtet. $\mathrm{Da}$ es meine Hauptaufgabe war zu sehen, wie überhaupt die Reihen konvergieren, und Aufschlüsse darüber zu bekommen, wie sich die Sache stellen würde in Fällen, wo man mit größeren Schwierigkeiten zu tun hätte, habe ich die Rechnung viel weitläufiger ausgefuhrt als es für diesen Fall nötig gewesen wäre.

Wenn wir die Bezeichnungsweise von Oppolzer (Bahnbestimmung II, 235-6) wählen, haben wir die folgenden Ausdrücke in Reihen zu entwickeln: $r \sin u, r \cos u, r \sin v$, $\sin v, \cos v, r, \mathrm{I} / r, \cos E, \xi_{1}, \eta_{1}, \zeta_{1}$. Diese Größen lassen sich leicht mit Hilfe der folgenden Formeln aus den schon bekannten Entwickelungen für $x, y, z$ und $x_{1}, y_{1}, z_{1}$ herleiten: 


$$
\begin{array}{rlrl}
r & =\sqrt{x^{2}+y^{2}+z^{2}}, & \sin v=\frac{r \sin v}{r}, \\
r \sin u & =\frac{z}{\sin i}, & \cos v=\frac{r \cos v}{r}, \\
r \cos u & =x \cos \Omega+y \sin \delta, \quad \cos u=\frac{r \cos u}{r}, & \cos E=\frac{r \cos v}{a}+e, \\
r \sin v & =r \sin u \cos \omega-r \cos u \sin \omega, \\
r \cos v & =r \cos u \cos \omega+r \sin u \sin \omega, \\
\xi_{1} & =x_{1}\left(\frac{x}{r}\right)+y_{1}\left(\frac{y}{r}\right)+z_{1}\left(\frac{z}{r}\right), \\
\eta_{1} & =-x_{1}\left(\cos u \cos i \sin \delta+\sin u \cos \Omega_{3}+y_{1}(\cos u \cos i \cos \Omega-\sin u \sin \delta)+z_{1} \cos u \sin i,\right. \\
\zeta_{1} & =x_{1} \sin i \sin \Omega-y_{1} \sin i \cos \Omega+z_{1} \cos i .
\end{array}
$$

Wenn wir diese Rechnungen ausführen und in die Differentialgleichungen der Elementenstörungen einsetzen, erhalten wir, unter selbstverständlicher Vernachlässigung der Sonnenstörungen, (in Längenmaß):

$$
\begin{aligned}
& \frac{\mathrm{d} i}{\mathrm{~d} \tau}=m_{1}[2.91010]\left(\frac{\rho_{0}}{\rho}\right)^{3}\left(+0.02258-0.14 \mathrm{I} 57 \cdot \tau+0.000150 \cdot \tau^{2}+0.00007215 \cdot \tau^{3}-0.000000059 \cdot \tau^{4}\right) \\
& \frac{\mathrm{d} \Omega}{\mathrm{d} \tau}=m_{1}[2.91010]\left(\frac{\rho_{0}}{\rho}\right)^{3}(-0.00009+0.00388-0.020782-0.00000200+0.000005324) \\
& \frac{\mathrm{d} \mu}{\mathrm{d} \tau}=m_{1}[1.37400 \mathrm{n}]\left(\frac{\rho_{0}}{\rho}\right)^{3}(-0.03840-0.01529-0.000366+0.00000737+0.000000022) \\
& \frac{\mathrm{d} L_{1}}{\mathrm{~d} \tau}=m_{1}[2.91010]\left(\frac{\varrho_{0}}{\rho}\right)^{3}(+0.16390-0.08606+0.002692+0.00001050+0.000000317) \\
& \frac{\mathrm{d} \pi}{\mathrm{d} \tau}=m_{1}[2.91010]\left(\frac{\rho_{0}}{\rho}\right)^{3}(+2.19773+1.7628 \mathrm{r}+0.027072-0.00010016-0.00001972 \mathrm{I}) \\
& \frac{\mathrm{d} \varphi}{\mathrm{d} \tau}=m_{1}[2.9 \mathrm{r010}]\left(\frac{\varphi_{0}}{\rho}\right)^{3}(+0.07428+0.0104 \mathrm{r}-0.000080-0.00002056+0.000000060)
\end{aligned}
$$

wo zu beachten ist, daß die Differentialkoeffizienten nach $\tau \mid$ wir sofort, daß alle Integrale von der Form (nicht nach $t$ ) genommen sind.

Nach der Ausführung der nötigen Multiplikation haben wir mit einer Reihe von Integralen zu tun, die von der Form

$$
\int \frac{\tau^{p} d \tau}{\left(1+\tau^{2}\right)^{1 / 2(2 n+1)}}
$$

sind. Diese Integrationen lassen sich alle in geschlossener Form ausfüren*). Wenn wir die Integrationsgrenzen symmetrisch um die Zeit der größten Annäherung wählen, sehen

$$
\begin{aligned}
\int \frac{\mathrm{d} \tau}{\left(\mathrm{I}+\tau^{2}\right)^{3 / 2}}=\frac{\tau}{\sqrt{\mathrm{I}+\tau^{2}}} ; & \int \frac{\tau^{4} \mathrm{~d} \tau}{\left(\mathrm{I}+\tau^{2}\right)^{8 / 2}}=\frac{\tau\left(3+\tau^{2}\right)}{2 \sqrt{\mathrm{I}+\tau^{2}}-3 / 2 \log \left(\tau+\sqrt{\mathrm{I}+\tau^{2}}\right) ;} \\
\int \frac{\tau^{2} \mathrm{~d} \tau}{\left(1+\tau^{2}\right)^{8 / 2}}=-\frac{\tau}{\sqrt{\mathrm{I}+\tau^{2}}+\log \left(\tau+\sqrt{\mathrm{I}+\tau^{2}}\right) ;} & \int \frac{\tau^{4} \mathrm{~d} \tau}{\left(1+\tau^{2}\right)^{5 / 2}}=-\frac{\tau\left(3+4 \tau^{2}\right)}{3\left(1+\tau^{2}\right)^{3 / 2}}+\log \left(\tau+\sqrt{\mathrm{I}+\tau^{2}}\right) .
\end{aligned}
$$

In unserem Falle brauchen wir nur das erste Glied in Betracht zu ziehen. Wenn wir fưr die Masse des Planeten (386) das bei Bauschinger (Tafel X) angegebene hypothetische Volumen annehmen, also in Einheiten der Sonnenmasse:

$$
m_{1}=[8.93-20] \text {, }
$$

so erhalten wir durch die Integration von -30 Tage bis +30 Tage für alle Elemente Störungswerte, die weit unter-

halb der wahrscheinlichen Fehler der Elemente liegen. Für die Störung der mittleren Bewegung ergibt sich z. B.

$$
\Delta \mu=+0.0000032 \text {. }
$$

Für die in Frage stehende Annäherung der Planeten (366) und (386) hat also die Störungsrechnung ein negatives Resultat gegeben. Man sieht aber auch sofort, daß im System

*) Für die doppelte Integration $\iint \frac{\mathrm{d} \mu}{\mathrm{d} \tau} \mathrm{d} \tau^{2}$ wird bei den höheren Gliedern die Sache komplizierter. 
der kleinen Planeten sehr wohl Fälle vorkommen können und aller Wahrscheinlichkeit nach auch vorkommen werden, wo eine merkbare, wenn auch nicht beträchtliche Störung durch eine ähnliche Annäherung hervorgerufen wird (vergl. z. B. A. Galles Übersicht S. 50-52 seiner Abhandlung $\gg$ Zur Berechnung der Proximitäten von Asteroiden-Bahnenk).

In unserem Falle lagen die Verhältnisse für die numerische Rechnung sehr günstig. Die Annäherung ist so unbedeutend, daß auch bei einem viel größeren Massenwerte die Berechnung des ersten Gliedes genügt hätte. Wenn in einem anderen Falle die kleinste Distanz viel kleiner wird, könnte man zur Berechnung von mehr Gliedern gezwungen werden.

Außerdem gibt es einen anderen Umstand, der die Rechnung vereinfacht hat. Die Annäherung ist in diesem Falle dadurch entstanden, daß die zwei Planeten nahe gleichzeitig den einen Schnittpunkt ihrer Bahnebenen passiert haben. Da die gegenseitige Bahnneigung ziemlich beträchtlich ist, sind die zwei Planeten aneinander relativ schnell vorübergegangen. Annäherungen können aber auch derart sein, daß die beiden Bahnen nicht so schnell wieder auseinander laufen, und in einem solchen Falle könnte es leicht vorkommen, daß die zwei Körper längere Zeit in einer beträchtlichen Annäherung verweilten, wovon die Folge wäre, daß man die Integrationsgrenzen beträchtlich erweitern müßte.

Es könnte uberhaupt in Frage gestellt werden, ob nicht solche Fälle möglich wären, wo in der Entwickelung von $1 / \varrho^{3}$ die Nenner $\left(1+\tau^{2}\right)^{1 / 2}(2 n+1)$ durch solche von der Form $\left(\mathrm{I}+a \tau^{2}+b \tau^{3}+c \tau^{4}\right)^{1 / 2(2 n+1)}$ ersetzt werden müßten, wo wir also bei der Integration mit elliptischen Integralen zu tun hätten. Man könnte sich z. B. einen der. Fig. I entsprechenden Fall denken, nämlich den einen Körper in einer wenig exzentrischen, den anderen in einer stark exzentrischen Bahn in derselben Richtung laufend, und $z$ war so, daß sich die beiden Bahnen in kurzer Zeit zweimal schnitten. Wenn die zwei Körper gleichzeitig in $A$ resp. $B$ eintreffen und die Geschwindigkeiten nahe gleich groß sind, würden wir bei $A B$ ein Maximum der Distanz, in der Nähe von $C$ und $D$ zwei Minima haben. Dann würden in der Entwickelung von $\varrho^{2}$ bis zur vierten Potenz von $\tau$ die Koeffizienten von derselben Größenordnung sein. Setzen wir der Einfachheit wegen vollständige Symmetrie voraus, so hätten wir:

$$
\rho^{2}=\rho_{0}^{2}\left(1-\tau^{2}+a \tau^{4}+\cdots\right),
$$

wo $a$ von derselben Größenordnung wie $I$ ist und die folgenden Koeffizienten schnell abnehmen. Fig. 2 gibt mit $\tau$

Kiel, Bureau der Astr. Nachr., I 904 Januar. als Abszisse den Wert von $\left(\frac{\varrho}{\rho_{0}}\right)^{2}$, wenn wir $a=$ I setzen und die folgenden Koeffizienten vernachlässigen.

Ganz allgemein könnte man sich ja ein solches Problem zur Lösung stellen. In unserem Sonnensystem, oder überhaupt wo die zwei Körper der Anziehung einer überwiegenden Zentralkraft unterworfen sind, dürfte aber ein solcher Fall ausgeschlossen sein, und zwar aus folgendem Grunde.

Wenn die zwei. Bahnen in kurzer Zeit, und nur von relativ kurzen Zeiten ist überhaupt die Rede, sich zweimal

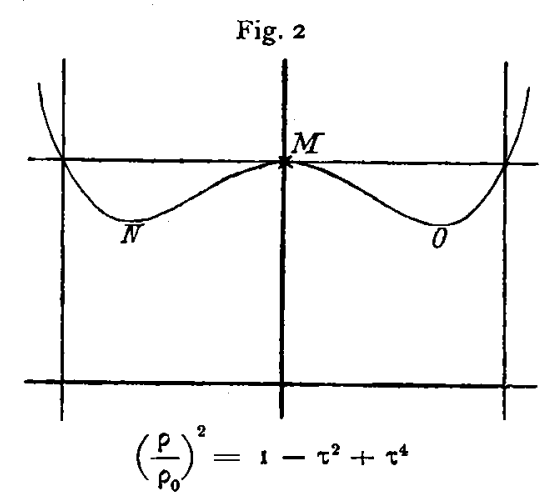
schneiden sollen, ist es eine Bedingung, daß der Krümmungsunterschied der Bahnen groß ist. Wenn aber der Krümnungsunterschied groß ist, muß sich der in der weniger gekrümmten Bahn laufende Körper zu der Zeit mit viel größerer Geschwindigkeit bewegen als der andere, weil sonst die nach der

Zeit genommenen zweiten Differentialkoeffizienten der Ko. ordinaten für beide Körper nicht nahe gleich groß sein könnten, was aber, laut den Gleichungen

$$
\frac{\mathrm{d}^{2} x_{1}}{\mathrm{~d} t^{2}}=\frac{k^{2} x_{1}}{r_{1}^{3}} ; \quad \frac{\mathrm{d}^{2} x}{\mathrm{~d} t^{2}}=\frac{k^{2} x}{r^{3}} \text { etc. }
$$

der Fall sein muß, wenn die Koordinaten selbst nahe gleich sind.

Wenn aber die Geschwindigkeiten sehr verschieden sind, kommen sich die zwei Körper in den Punkten $C$ und $D$ überhaupt nicht mehr nahe, d. i. wir haben in $C$ und $D$ keine Distanzminima mehr und in $A B$ kein Maximum, sondern ein Minimum.

Mit anderen Worten und etwas allgemeiner ausgedrückt:

Wenn wir von solchen Făllen absehen, wo wegen fast völliger Identität der beiden Bahnen die Annäherung der Körper sehr lange Zeit dauert, können in den Entwickelungen der Koordinatendifferenzen die konstanten und die der Zeit proportionalen Glieder nicht gleichzeitig im Vergleich mit den entsprechenden Werten in den Koordinatenreihen sehr klein sein. Wenn eine Annäherung besteht, werden also die mit $\tau$ multiplizierten Glieder groß im Verhältnis zu den mit $\tau^{2}, \tau^{3}$ etc. multiplizierten, was zur Folge hat, daß in der Entwickelung von $\rho^{2}$ die Glieder mit $\tau^{3}$ etc. dem Glied $\tau^{2}$ gegenüber klein sind und daß $1 / \varrho^{3}$ also für die Dauer einer Annäherung von zwei Planeten immer in der Form

$$
\sum \sum A_{p, n} \frac{\tau^{p}}{\left(\mathrm{I}+\tau^{2}\right)^{1 / 2(2 n+\mathrm{I})}}
$$

entwickelt werden kann.

Elis Strömgren.

\section{Bedeckung des Aldebaran 1903 0kt. 10.}

Eintritt: Pokrowski $10^{\mathrm{h}} 14^{\mathrm{m}} 10^{\mathrm{s}} 6 \mathrm{M}$ M. Z. Jurj., Scharbe $10^{\mathrm{h}} 14^{\mathrm{m}} 10^{\mathrm{s}} \cdot 4 ;$ Austritt: Pokrozeski $\mathrm{I1}^{\mathrm{h}}{ }_{1} 3^{\mathrm{m}} 43^{\mathrm{s}} \cdot 4$, Scharbe $11^{\mathrm{h}} 13^{\mathrm{m}} 43^{\mathrm{s}} \cdot 4$. Jurjew - Dorpat, 1903 Nov. 27.

K. Pokroweski, S. Scharbe. 